\title{
GENERALIZATION OF TWO RESULTS OF THE THEORY OF UNIFORM DISTRIBUTION
}

\author{
PETKO D. PROINOV
}

AbStract. For a sequence $x_{1}, \ldots, x_{N}$ of points in $[0,1]$ and a sequence $p_{1}, \ldots, p_{N}$ $\left(p_{1}+p_{2}+\cdots+p_{N}=1\right)$ of nonnegative numbers, define the distribution function

$$
g(x)=x-\sum_{x_{k}<x} p_{k} .
$$

Let $\varphi$ be an increasing function on $[0,1]$ and $\varphi(0)=0$. The main result of the paper is

$$
F\left(D_{N}\right) \leqslant \int_{0}^{1} \varphi(|g(x)|) d x \leqslant \varphi\left(D_{N}\right),
$$

where $D_{N}$ is the supremum norm of $g$ on $[0,1]$ and $F$ is the antiderivative of $\varphi$ with $F(0)=0$. This result generalizes and improves an estimate of Niederreiter [1] for the $L^{2}$ discrepancy of the sequence $x_{1}, \ldots, x_{N}$. Applying the above inequality we also obtain a new criterion for uniform distribution modulo one.

1. Introduction. Let us recall some definitions and results of the theory of uniform distribution modulo one.

Definition A. Let $x_{1}, x_{2}, \ldots, x_{N}$ be a finite sequence in the interval $[0,1]$. The number

$$
D_{N}^{(p)}=\left(\int_{0}^{1}\left|x-\frac{1}{N} \sum_{\substack{1 \leqslant k \leqslant N \\ x_{k}<x}} 1\right|^{p} d x\right)^{1 / p}, \quad 0<p \leqslant \infty,
$$

is called the $L^{p}$ discrepancy of the given sequence.

In what follows, we shall write $D_{N}$ instead of $D_{N}^{(\infty)}$.

H. Niederreiter proved the following theorem using the well-known inequality of LeVeque.

Theorem A (Niederreiter [1]). For any sequence $x_{1}, x_{2}, \ldots, x_{N}$ in $[0,1]$ we have

$$
\frac{1}{\sqrt{12}} D_{N}^{3 / 2} \leqslant D_{N}^{(2)} \leqslant D_{N}
$$

Definition B. Let $\sigma=\left(x_{n}\right)$ be an infinite sequence in $[0,1]$. For the infinite sequence $\sigma$, the $L^{p}$ discrepancy $D_{N}^{(p)}(\sigma)$ is defined to be the $L^{p}$ discrepancy of the initial segment formed by the first $N$ terms of $\sigma$.

Again, we shall write $D_{N}(\sigma)$ instead of $D_{N}^{(\infty)}(\sigma)$.

Received by the editors August 20, 1984 and, in revised form, January 15, 1985.

1980 Mathematics Subject Classification. Primary 10K05, 10F40. 
Definition C. An infinite sequence $\sigma$ in $[0,1]$ is said to be uniformly distributed (in the sense of Weyl) if

$$
\lim _{N \rightarrow \infty} D_{N}(\sigma)=0
$$

The following criterion for uniform distribution is well known.

TheOREM B (SoBol [2, P. 115]). Suppose $0<p<\infty$. Then an infinite sequence $\sigma$ in $[0,1]$ is uniformly distributed if and only if

$$
\lim _{N \rightarrow \infty} D_{N}^{(p)}(\sigma)=0
$$

In $\S 2$ we generalize and improve Theorem A. In $\S 3$ we generalize Theorem B.

2. Generalization of Theorem A. Suppose we are given a finite sequence $x_{1}$, $x_{2}, \ldots, x_{N}$ in $[0,1]$ and a finite sequence $p_{1}, p_{2}, \ldots, p_{N}$ of nonnegative numbers. We call the numbers $p_{1}, p_{2}, \ldots, p_{N}$ weights of the numbers $x_{1}, x_{2}, \ldots, x_{N}$, respectively. Let us define the functions $h$ and $g$ on $[0,1]$ by

$$
h(x)=\sum_{\substack{1 \leqslant k \leqslant N \\ x_{k}<x}} p_{k}
$$

and

$$
g(x)=x-h(x) \text {. }
$$

Obviously, the function $h$ is increasing on $[0,1]$ and the function $g$ is piecewise linear on $[0,1]$.

DEFINITION 1. The number

$$
D_{N}^{(p)}=\left(\int_{0}^{1}|g(x)|^{p} d x\right)^{1 / p}, \quad 0<p \leqslant \infty,
$$

is said to be the $L^{p}$ discrepancy of the sequence $x_{1}, x_{2}, \ldots, x_{N}$ with respect to the weights $p_{1}, p_{2}, \ldots, p_{N}$.

Instead of $D_{N}^{(\infty)}$ we shall write $D_{N}$. Evidently,

$$
D_{N}=\sup _{0 \leqslant x \leqslant 1}|g(x)| \text {. }
$$

Comparing Definition A with Definition 1 we see that the $L^{p}$ discrepancy of the sequence $x_{1}, x_{2}, \ldots, x_{N}$ is equal to the $L^{p}$ discrepancy of this sequence with respect to the weights $p_{1}=p_{2}=\cdots=p_{N}=1 / N$.

THEOREM 1. Let $\varphi$ be an increasing function on $[0,1], \varphi(0)=0$ and

$$
F(x)=\int_{0}^{x} \varphi(t) d t .
$$

Then for any sequence $x_{1}, x_{2}, \ldots, x_{N}$ in $[0,1]$ and any weights $p_{1}, p_{2}, \ldots, p_{N}$ with

$$
\sum_{k=1}^{N} p_{k}=1
$$

we have

$$
F\left(D_{N}\right) \leqslant \int_{0}^{1} \varphi(|g(x)|) d x \leqslant \varphi\left(D_{N}\right),
$$

where the function $g$ is defined by (3). 
Proof. The second inequality in (7) is obvious. It holds true because $\varphi$ increases on $[0,1]$. Now, we shall prove the first inequality in (7). Let $a$ be an arbitrary real number with

$$
0<a<D_{N}
$$

First we shall prove the following inequality.

$$
F(a) \leqslant \int_{0}^{1} \varphi(|g(x)|) d x .
$$

It follows from (4) and (8) that there exists a number $x_{0} \in[0,1]$ such that

$$
\left|g\left(x_{0}\right)\right|>a \text {. }
$$

According to the definition of $g$, the above inequality can be written as

$$
\left|x_{0}-h\left(x_{0}\right)\right|>a \text {. }
$$

Hence, there are two possible cases:

$$
h\left(x_{0}\right)>x_{0}+a \text { or } h\left(x_{0}\right)<x_{0}-a .
$$

Further, we shall prove (9) in the first case only because it can similarly be proved in the second case as well.

Suppose $h\left(x_{0}\right)>x_{0}+a$. Then from (2) and (6), we have

$$
\left[x_{0}, x_{0}+a\right] \subset[0,1] \text {. }
$$

Since $\varphi$ is an increasing function on $[0,1]$ and $\varphi(0)=0$, it follows that the inequality $\varphi(|g(x)|) \geqslant 0$ holds for every $x \in[0,1]$. Hence, we obtain from (10)

$$
\int_{0}^{1} \varphi(|g(x)|) d x \geqslant \int_{x_{0}}^{x_{0}+a} \varphi(|g(x)|) d x .
$$

Now suppose that $x \in\left[x_{0}, x_{0}+a\right]$. Since the function $h$ increases on $[0,1]$, we deduce

$$
g(x) \leqslant x-h\left(x_{0}\right)<x-x_{0}-a \leqslant 0 .
$$

Therefore,

$$
|g(x)|=-g(x)>x_{0}+a-x .
$$

Hence, using (11) and (5) we get

$$
\int_{0}^{1} \varphi(|g(x)|) d x \geqslant \int_{x_{0}}^{x_{0}+a} \varphi\left(x_{0}+a-x\right) d x=F(a) .
$$

Thus, (9) is proved in the first case.

Now it follows from (9) that

$$
\sup _{0<a<D_{N}} F(a) \leqslant \int_{0}^{1} \varphi(|g(x)|) d x .
$$

But the function $F$ is increasing on $[0,1]$ because $\varphi(x) \geqslant 0$ for every $x \in[0,1]$. Hence, $F$ is increasing on $\left[0, D_{N}\right]$, too, because $0<D_{N} \leqslant 1$. Therefore,

$$
\sup _{0<a<D_{N}} F(a)=F\left(D_{N}\right)
$$

Finally, the first inequality in (7) follows from (12) and (13). Theorem 1 is proved. 
Setting $\varphi(x)=x^{p}(0<p<\infty)$ in Theorem 1 , we immediately obtain

Corollary 1. Suppose $0<p<\infty$. Then for any sequence $x_{1}, x_{2}, \ldots, x_{N}$ in $[0,1]$ and any weights $p_{1}, p_{2}, \ldots, p_{N}$ with (6) we have

$$
\frac{1}{(p+1)^{1 / p}} D_{N}^{1+1 / p} \leqslant D_{N}^{(p)} \leqslant D_{N} .
$$

REMARK 1. From (14) we get the following estimate for $p=2$,

$$
\frac{1}{\sqrt{3}} D_{N}^{3 / 2} \leqslant D_{N}^{(2)} \leqslant D_{N} .
$$

It is easy to see that (15) improves estimate (1) of Niederreiter.

REMARK 2 . The first inequality in (7) changes into an equality if $x_{1}=x_{2}=\cdots=$ $x_{N}=0$.

3. Generalization of Theorem B. Suppose we are given two infinite triangular matrices $X=\left(x_{k}^{(n)}\right)$ and $P=\left(p_{k}^{(n)}\right)$ with $0 \leqslant x_{k}^{(n)} \leqslant 1$ and $p_{k}^{(n)} \geqslant 0(n=1,2, \ldots$; $k=1, \ldots, n)$. We call the matrix $P$ a weight matrix of the matrix $X$.

Definition 2. Suppose $0<p \leqslant \infty$. The $L^{p}$ discrepancy $D_{n}^{(p)}(X, P)$ is defined to be the $L^{p}$ discrepancy of the sequence $x_{1}^{(n)}, x_{2}^{(n)}, \ldots, x_{n}^{(n)}$ with respect to the weights $p_{1}^{(n)}, p_{2}^{(n)}, \ldots, p_{n}^{(n)}$, i.e.

$$
D_{n}^{(p)}(X, P)=\left(\int_{0}^{1}\left|g_{n}(x)\right|^{p} d x\right)^{1 / p},
$$

where

$$
g_{n}(x)=x-\sum_{\substack{1 \leqslant k \leqslant n \\ x_{k}^{(n)}<x}} p_{k}^{(n)} .
$$

Definition 3 (SEE [3]). The matrix $X$ is said to be uniformly distributed with respect to the weight matrix $P$ if

$$
\lim _{n \rightarrow \infty} D_{n}(X, P)=0 \text {. }
$$

Definition 4. Let $\varphi$ be a function defined on $[0,1]$. We call $\varphi$ a basic function if it satisfies the following three conditions:

(i) $\varphi$ is increasing on $[0,1]$,

(ii) $\lim _{x \rightarrow 0^{+}} \varphi(x)=0$,

(iii) $\varphi(x)=0$ if and only if $x=0$.

LemMa 1. Let $\varphi$ be a basic function. Then the function $F$ defined by (5) is a basic function as well.

Proof. From (5), (i) and (iii), we deduce

$$
F\left(x_{2}\right)-F\left(x_{1}\right) \geqslant \frac{x_{2}-x_{1}}{2} \varphi\left(\frac{x_{1}+x_{2}}{2}\right)>0
$$

for all $x_{1}$ and $x_{2}$ with $0 \leqslant x_{1}<x_{2} \leqslant 1$. Therefore, $F$ is strictly increasing on [0,1]. Hence, for every $x \in(0,1]$ we have

$$
0=F(0)<F(x) \leqslant x \varphi(x) .
$$

Passing to the limit as $x \rightarrow 0^{+}$in this inequality, we get $\lim _{x \rightarrow 0^{+}} F(x)=0$. 
LeMma 2. Let $\varphi$ be a basic function. Then the matrix is uniformly distributed with respect to the weight matrix if and only if

$$
\lim _{n \rightarrow \infty} \varphi\left(D_{n}(X, P)\right)=0 .
$$

Proof. The necessity follows immediately from (ii) and Definition 3. Now suppose that (19) holds, but (18) does not hold. Then there exists a positive number $\varepsilon_{0}$ such that the inequality

$$
D_{n}(X, P) \geqslant \varepsilon_{0}
$$

holds for infinitely many values of $n$. It follows from (20) and (i) that

$$
\varphi\left(D_{n}(X, P)\right) \geqslant \varphi\left(\varepsilon_{0}\right) .
$$

From (19), (21) and (iii), we deduce

$$
0=\lim _{n \rightarrow \infty} \varphi\left(D_{n}(X, P)\right) \geqslant \varphi\left(\varepsilon_{0}\right)>0
$$

which is a contradiction. Therefore, if (19) holds then (18) holds, too, i.e. $X$ is uniformly distributed with respect to $P$.

The following criterion for uniform distribution is a generalization of Theorem $\mathrm{B}$.

THEOREM 2. Let $\varphi$ be a basic function and let $P$ be a weight matrix with

$$
\sum_{k=1}^{n} p_{k}^{(n)}=1 \quad(n=1,2, \ldots) .
$$

Then a matrix $X$ is uniformly distributed with respect to the weight matrix $P$ if and only if

$$
\lim _{n \rightarrow \infty} \int_{0}^{1} \varphi\left(\left|g_{n}(x)\right|\right) d x=0
$$

where $g_{n}(x)$ is defined by (17).

Proof. By Theorem 1 we have

$$
F\left(D_{n}(X, P)\right) \leqslant \int_{0}^{1} \varphi\left(\left|g_{n}(x)\right|\right) d x \leqslant \varphi\left(D_{n}(X, P)\right) .
$$

Since $\varphi$ is a basic function, it follows from Lemma 1 that $F$ is a basic function, too. Now, the assertion follows from (23) and Lemma 2.

Setting $\varphi(x)=x^{p}(0<p<\infty)$ in Theorem 2 , we immediately obtain

COROllary 2. Let $0<p<\infty$ and $P$ be a weight matrix with (22). Then a matrix $X$ is uniformly distributed with respect to the weight matrix $P$ if and only if

$$
\lim _{n \rightarrow \infty} D_{n}^{(p)}(X, P)=0 .
$$

REMARK 3. It is easy to see that Corollary 2 is a generalization of Theorem $\mathrm{B}$. Indeed, let $\sigma=\left(x_{n}\right)$ be an infinite sequence in [0,1]. Applying Corollary 2 for the matrices $X=\left(x_{k}^{(n)}\right)$ and $P=\left(p_{k}^{(n)}\right)$ with $x_{k}^{(n)}=x_{k}$ and $p_{k}^{(n)}=1 / n(n=1,2, \ldots$; $k=1, \ldots, n)$ we get Theorem $\mathrm{B}$. 
4. Final remark. Theorem 2 shows that as a measure of the distribution of a matrix $X$ with respect to a weight matrix $P$, alongside with the $L^{p}$ discrepancy $D_{n}^{(p)}(X, P)$, one may use the $\varphi$-discrepancy

$$
D_{n}^{(\varphi)}(X, P)=\int_{0}^{1} \varphi\left(\left|g_{n}(x)\right|\right) d x
$$

where $\varphi$ is a basic function and $g_{n}(x)$ is defined by (17).

Similarly, as a measure of the distribution of a sequence $x_{1}, x_{2}, \ldots, x_{N}$ in $[0,1]$ with respect to the weights $p_{1}, p_{2}, \ldots, p_{N}$, alongside with the $L^{p}$ discrepancy $D_{N}^{(p)}$, one can use the $\varphi$-discrepancy

$$
D_{N}^{(\varphi)}=\int_{0}^{1} \varphi(|g(x)|) d x
$$

where $\varphi$ is a basic function, too, and $g(x)$ is defined by (3).

\section{REFERENCES}

1. H. Niederreiter, Application of diophantine approximation to numerical integration, Diophantine Approximation and its Applications (C. F. Osgood, ed.), Academic Press, New York, 1973, pp. 129-199.

2. I. M. Sobol, Multidimensional quadrature formulae and Haar functions, Nauka, Moscow, 1969.

3. P. D. Proinov, Note on the convergence of the general quadrature process with positive weights, Constructive Function Theory'77 (Bl. Sendov and D. Vačov, eds.), Sofia, 1980, pp. 121-125.

Department of Mathematics, University of Plovdiv, 4000 Plovdiv, Bulgaria 